\title{
Direct evidence of Rabi oscillations and antiresonance in a strongly coupled organic microcavity
}

\author{
S. K. Rajendran, ${ }^{1}$ W. Wang, ${ }^{2}$ D. Brida,${ }^{1,3}$ A. De Sio, ${ }^{2}$ E. Sommer, ${ }^{2}$ R. Vogelgesang, ${ }^{2}$ D. Coles, ${ }^{4}$ \\ D. G. Lidzey, ${ }^{4}$ G. Cerullo, ${ }^{1}$ C. Lienau, ${ }^{2}$ and T. Virgili ${ }^{1, *}$ \\ ${ }^{1}$ Istituto di Fotonica e Nanotecnologie-CNR, Dipartimento di Fisica, Politecnico di Milano, \\ Piazza Leonardo Da Vinci 32, I-20133 Milano, Italy \\ ${ }^{2}$ Institut für Physik and Center of Interface Science, Carl von Ossietzky Universität Oldenburg, D-26111 Oldenburg, Germany \\ ${ }^{3}$ Department of Physics and Center for Applied Photonics, University of Konstanz, D-78457 Konstanz, Germany \\ ${ }^{4}$ Department of Physics and Astronomy, University of Sheffield, Hicks Building, Hounsfield Road, Sheffield S3 7RH, United Kingdom
}

(Received 16 December 2014; revised manuscript received 27 April 2015; published 28 May 2015)

\begin{abstract}
We report the direct observation of 30 -fs period Rabi oscillations between excitons and cavity photons in a strongly coupled $J$-aggregate microcavity by means of time-resolved up-conversion and spectral interferometry measurements. The time structure of the transmitted electric field, measured by linear spectral interferometry, shows pronounced ultrafast beats. Its spectral phase reveals a distinct signature caused by destructive interference between the coherent drive and the field radiated by the exciton. This antiresonance selectively probes the uncoupled exciton excitation, and its observation uncovers the coherent and ultrafast exchange of energy between the optically excited cavity and the $J$-aggregate excitons, as confirmed by transfer matrix calculations.
\end{abstract}

DOI: 10.1103/PhysRevB.91.201305

PACS number(s): 71.36.+c, 42.87.Bg, 78.47.D-

Cavity polaritons exhibit a wealth of fascinating phenomena, due to strong, coherent coupling between their photonic and excitonic components, such as the formation of Bose-Einstein condensates [1], polariton lasing [2,3], and polariton superfluidity [4]. Applications such as the polariton amplifier [5,6] and a polariton light-emitting diode $[7,8]$ have already been demonstrated. The microscopic origin of these phenomena lies in the coherent interaction between excitons and cavity photons, via coupling of the excitonic transition dipole moment to fluctuating (vacuum) electromagnetic fields stored within the cavity. This strong coupling results in the formation of new hybrid lower (LP) and upper (UP) excitonphoton polariton modes, separated in energy by normal mode splitting [9,10]. Polaritons are coherent superpositions of the bare exciton and cavity photon modes; when one of the pure components is driven, a periodic energy exchange between the excitonic and photonic components occurs in the form of Rabi oscillations. From a dynamical perspective, strong coupling is directly connected with a periodic flow of energy between excitons and cavity photons: The normal mode splitting reflects ultrafast Rabi oscillations between the excitonic and the photonic components of the system. Upon impulsive excitation, this coupling leads to characteristic interference beats in the light transmitted through the cavity. As such, one of the most direct ways to probe coupling dynamics is to study, in the time domain, the electric field emitted by the cavity.

The optical properties of inorganic microcavities have been intensively studied, with experimental evidence of Rabi oscillations [11,12], nonlinearities in polariton splitting [13], polariton scattering, and relaxation effects [14,15]. In these systems, however, due to small normal mode splitting energies, most applications are confined to low temperatures. The large oscillator strength of organic semiconductors, on the other hand, allows the observation of a strong-coupling regime at room temperature and with easy fabrication techniques, thus

*Email address: tvirgili@polimi.it opening up a wide range of applications for cavity polaritons [16-19]. Strongly coupled (5,6-dichloro-2-[[5,6-dichloro-1ethyl-3-(4-sulphobutyl)benzimidazol-2 ylidene]propenyl]-1ethyl-3-(4-sulphobutyl) benzimidazolium hydroxide, inner salt, sodium salt) (TDBC) cyanine dye $J$ aggregates in a microcavity have therefore been studied intensely for their linear optical properties [20], temperature-dependent emission properties [21,22], and interactions with molecular vibrations $[23,24]$. The role of the uncoupled exciton reservoir in the relaxation dynamics of the cavity polariton states has also been theoretically [25-27] and experimentally investigated [28,29]. However, direct time-domain studies of the strong-coupling dynamics are challenging since the normal mode splitting in these systems often exceeds several tens or even hundreds of meV, resulting in periods of Rabi oscillations shorter than $50 \mathrm{fs}$. Hence, advanced ultrafast optical techniques, providing time resolution in the 10-fs range or even below, are required for such studies. Indeed, only very recently have Rabi oscillations in TDBC $J$ aggregates coupled to surface plasmon modes been observed in real time [30,31].

In this Rapid Communication, we study the roomtemperature real-time dynamics of light transmission through a strongly coupled organic microcavity, comprising a $J$ aggregated cyanine dye coupled to a dielectric microcavity. Time-dependent up-conversion measurements display pronounced temporal modulations indicative of Rabi oscillations. Spectral interferometry (SI) measurements show distinct phase signatures characteristic of the strongly coupled modes and display an antiresonance between the UP and LP resonances. Taken together, these measurements give direct evidence for strong coupling in organic microcavities and shed light on the coherent, ultrafast energy exchange between exciton and cavity photons in the strong-coupling regime.

We dispersed the cyanine dye TDBC [see the chemical structure in the inset of Fig. 1(a)] in a gelatin matrix forming $J$ aggregates (see the Supplemental Material for more details on the cavity preparation [32]). Figure 1(a) shows absorption and photoluminescence spectra of TDBC, which peak at 585 and $590 \mathrm{~nm}$, respectively. Intermolecular coupling delocalizes 
(a)

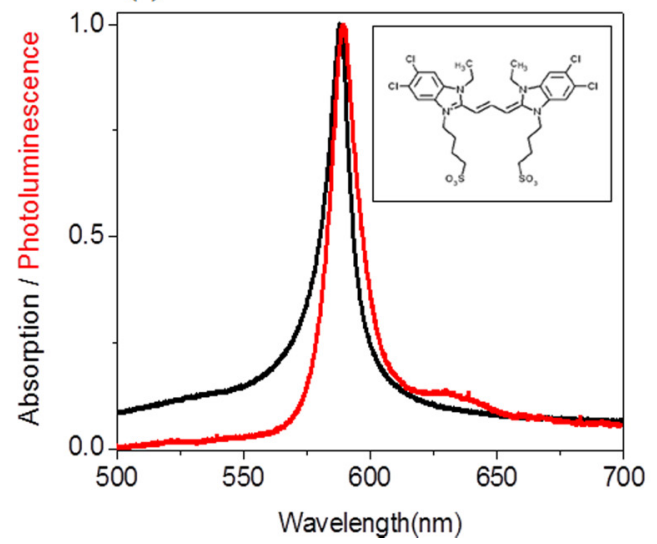

(b)

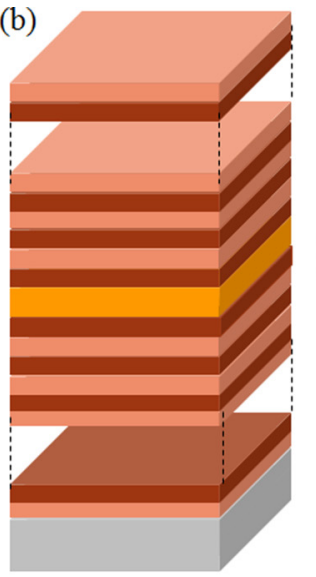

Distributed Bragg Reflector 2

Active material : J- Aggregate layer

Distributed Bragg

Reflector 1

Fused quartz substrate

FIG. 1. (Color online) (a) Absorption and pholuminescence spectra of the TDBC cyanine dye (chemical structure in the inset). (b) Schematic of the $J$-aggregate microcavity with the active material sandwiched between two distributed Bragg reflectors.

the excitation over many sites of the molecular aggregate, averaging over local inhomogeneities and leading to a spectral redshift and motional line narrowing [33]. The small Stokes shift, together with the large oscillator strength and the narrow linewidth of the $J$ aggregate, make this material an excellent candidate for exhibiting strong coupling between the exciton and a cavity mode.

The active TDBC layer of around $220 \mathrm{~nm}$ thickness was inserted between two distributed Bragg reflectors (DBRs), as shown in Fig. 1(b), forming a microcavity with its mode centered at $625 \mathrm{~nm}$ for normal incidence. The inset of Fig. 2(a) shows the experimental two-dimensional (2D) map of the normalized transmission through the microcavity upon angle tuning, with the intensity in the logarithmic scale. The expected cavity mode peak position, traced by a black solid line, shows, at an angle of $35^{\circ}$, a resonance with the exciton absorption (white solid line), forming the LP (blue line) and UP (red line) modes separated by a normal mode splitting energy of $140 \mathrm{meV}$. Due to the asymmetric line shape of the $J$-aggregate absorption peak, its tail partially overlaps with the UP mode, lowering the transmission of this peak. The angledependent cavity transmission was modeled with a transfer matrix (TM) approach, using the complex refractive index of the $J$ aggregate extracted from the absorption spectrum [34]. The simulation results (see Fig S1 in the Supplemental Material [32]) are in excellent agreement with experiments, although the spatial inhomogeneities of the DBR layer thicknesses make it difficult to exactly reproduce the sideband positions.

To directly probe the exciton-photon coupling in the microcavity in the time domain, we have temporally resolved the cavity transmission by ultrafast frequency up-conversion (see the Supplemental Material for details on the experimental setup [32]). In this experiment, the cavity is positively detuned by $5^{\circ}$ with respect to the resonance condition $\left(40^{\circ}\right.$ angle of incidence) in order to increase the transmission at the UP. An ultrashort broadband visible pulse, of $\approx 6$ fs duration, is incident on the microcavity. The spectrum of the transmitted light is displayed in Fig. 2(a) and shows the UP and LP peaks at 565 and $601 \mathrm{~nm}$, respectively, together with the cavity sidebands at $\approx 640$ and $525 \mathrm{~nm}$.
The temporal structure of the transmitted light is resolved by nonlinear gating with a time-delayed, bandwidth-limited 13-fs near-infrared pulse at $910 \mathrm{~nm}$ and recording the resulting sum-frequency spectra as a function of delay. The optical interaction with the microcavity is kept in the linear regime and nonlinear optics is only used to gate the temporal profile of the transmitted light. In the limit of infinitely short pump and gate pulses, the experiment therefore provides a measurement of the instantaneous intensity of the light transmitted through the microcavity, at the time defined by the delay between gate and pump pulse. The inset of Fig. 2(b) plots the experimental 2D up-conversion map, as a function of the detection wavelength and time delay; the maximum up-converted signal intensity is observed at $360 \mathrm{~nm}(3.46 \mathrm{eV})$. This detection energy is close to the sum of the average gate photon energy $(1.36 \mathrm{eV})$ and the LP (UP) resonance energies at $2.06 \mathrm{eV}(2.19 \mathrm{eV})$ and hence probes up-converted transmission from both the LP and UP modes. The up-conversion dynamics at a wavelength of $360 \mathrm{~nm}$, reported in the main panel of Fig. 2(b) as a solid line, displays a pronounced oscillatory modulation, with a period of $30 \pm 2 \mathrm{fs}(\approx 140 \mathrm{meV})$. This frequency closely matches the LP/UP normal mode splitting of $140 \mathrm{meV}$ seen in Fig. 2(a), and thus it seems natural to assign this oscillation to the interference between the transmitted UP and LP fields. The oscillatory signal displays a maximum around time zero, corresponding to a cosine-type modulation. It is superimposed on a background decaying with a time constant of $\sim 60$ fs that agrees very well with the spectral linewidth of the narrower LP mode. This background mainly stems from the up-conversion of the strong LP transmission. Similar oscillations, albeit with much longer periods, have been seen in earlier up-conversion measurements, and very recently also in pump-probe experiments on inorganic microcavities $[35,36]$. The results in Fig. 2(b) present a time-domain observation of such oscillations in an organic microcavity system.

Based on these up-conversion experiments, specifically the observation of a cosine-type modulation, we can conclude that there is a negligible phase shift between the fields transmitted through the LP and UP resonances. These results, however, do not yet allow one to decide whether these oscillations result from the coherent coupling between excitons and cavity 

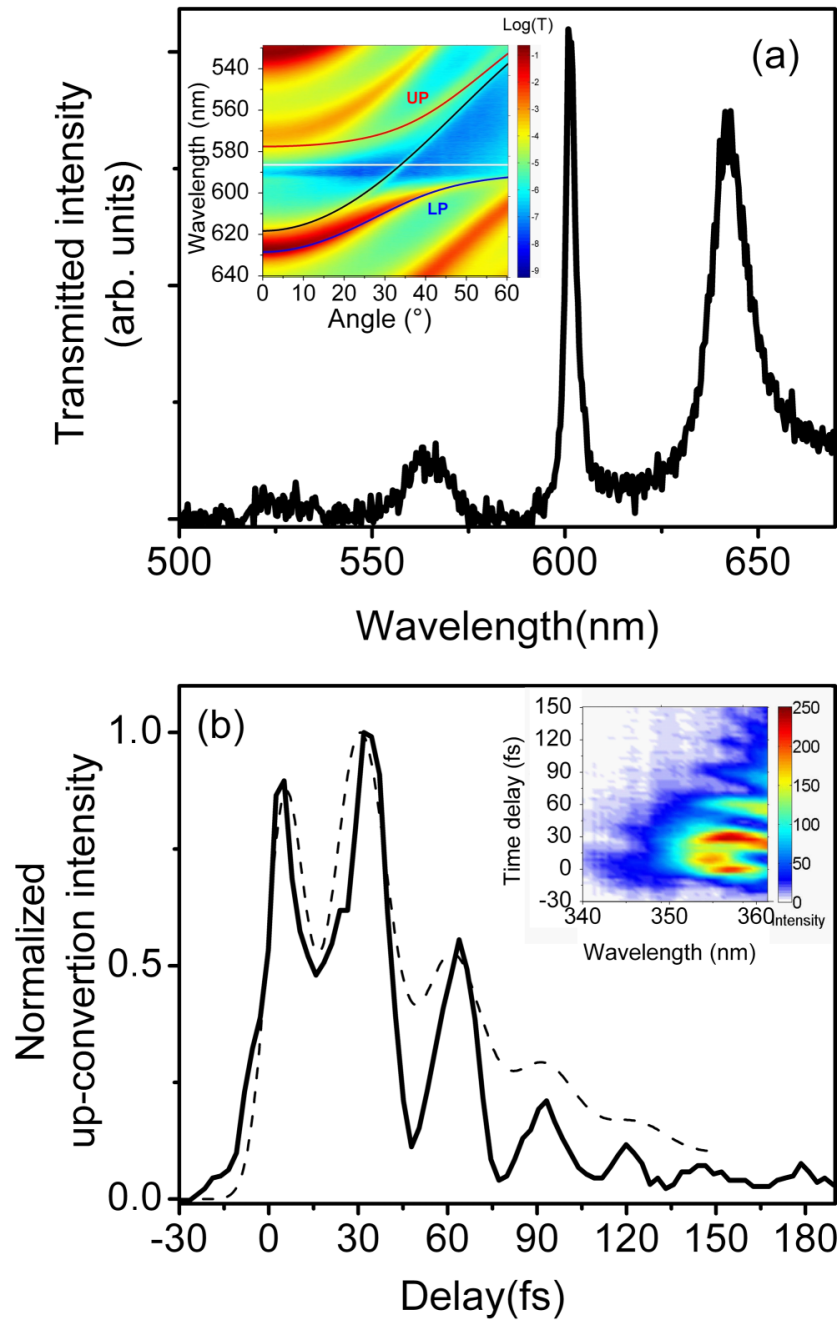

FIG. 2. (Color online) (a) Spectrum of the 6-fs pump pulse with a fluence of $3 \mathrm{~nJ} / \mathrm{cm}^{2}$, centered at around $600 \mathrm{~nm}$, transmitted through the microcavity at $40^{\circ}$ angle of incidence. In the inset, experimentally measured normalized transmission spectra are shown on a logarithmic scale as a function of incidence angle. The spectra show the anticrossing of the cavity polariton modes [blue (LP) and red (UP) solid lines], together with the exciton absorption (white solid line) and the dispersion of the empty cavity mode (black solid line). (b) Temporal dynamics of the up-converted signal detected at $360 \mathrm{~nm}$ (black line). Pronounced oscillations with a period of $\sim 30 \mathrm{fs}$, reflecting the interference between UP and LP transmission, are seen. The oscillations are superimposed on a background decaying monoexponentially with a rate $1 / \tau=1 / 60 \mathrm{fs}$. The dashed line is a simulation of the up-converted signal dynamics at a $360 \mathrm{~nm}$ detection wavelength. In the inset, a two-dimensional map of the time-resolved up-converted signal is shown as a function of the detection wavelength.

photons or if they simply reflect the interference between two uncoupled modes.

To account for the origin of the observed oscillations, we have measured the complex transmission spectrum $t(\omega)=$ $|t(\omega)| e^{i \varphi(\omega)}$ of the microcavity by means of angle-resolved SI $[37,38]$, which allows one to extract both the amplitude and the phase of the linear transmission spectrum. In SI, light from a broadband coherent source, in our case a white light supercontinuum, is split by a Mach-Zehnder interferometer into a sample and a reference beam. The sample beam is transmitted through the microcavity, whereas the reference beam traverses a quartz substrate. Both beams, after a suitable delay, are collinearly overlapped and sent to a spectrometer. Their interference spectrum exhibits (nearly) equidistant fringes in the frequency domain, with a spacing which is inversely proportional to their delay. The measurement of the shift of the fringe peaks between the data taken with the cavity and those taken with just the substrate on the sample beam gives the phase shift introduced by transmission through the microcavity. Due to the large linear phase introduced by the substrate, it is not possible to extract the absolute phase, but only a relative phase shift.

Figure 3(a) shows the microcavity intensity transmission $T(\omega)=|t(\omega)|^{2}$ at $40^{\circ}$ incidence. The UP and LP modes are at 563 and $600 \mathrm{~nm}$, respectively, whereas the sidebands of the cavity are positioned at 525 and $640 \mathrm{~nm}$. Figure $3(\mathrm{~b})$ shows the spectral phase $\varphi(\omega)$ extracted from the SI measurement. We observe clear phase jumps of $\pi$ at each of the four resonances (UP, LP, and the two sidebands). The UP and LP resonances are in phase with each other, as already anticipated by the up-conversion results. In addition, we find a distinct phase jump by $-\pi$ at a wavelength of $585 \mathrm{~nm}$, in between the LP and UP, in correspondence to the peak of the bare TDBC exciton. This negative phase jump is the signature of an antiresonance. Such antiresonances are well known in coupled mechanical oscillator systems but have been observed only very recently in a strongly coupled single-atom cavity system [39].

To understand the observed phase behavior, we compare our experimental data to a simple quantum model for the cavity transmission in the strong-coupling limit. This model considers a cavity mode coupled to an excitonic mode and assumes that the driving field is coupled only to the cavity mode. The cavity transmission can then be written as (see the Supplemental Material for the derivation of this expression [32])

$$
t(\omega)=\frac{i\left(\omega-\tilde{\omega}_{X}\right)}{\left(\omega-\tilde{\omega}_{\mathrm{LP}}\right)\left(\omega-\tilde{\omega}_{\mathrm{UP}}\right)} .
$$

Here, $\tilde{\omega}_{X, C}=\omega_{X, C}-i \gamma_{X, C}$ and $\tilde{\omega}_{\mathrm{LP}, \mathrm{UP}}=\frac{1}{2}\left(\tilde{\omega}_{X}+\tilde{\omega}_{C}\right) \pm$ $\sqrt{\left(\frac{\tilde{\omega}_{X}+\tilde{\omega}_{C}}{2}\right)^{2}-\left|\Omega_{R}\right|^{2}}$. The frequencies of the uncoupled exciton and cavity resonances are denoted as $\omega_{X}$ and $\omega_{C}$ and $\gamma_{X}=T_{2 X}^{-1}$ and $\gamma_{C}=T_{2 C}^{-1}$ are their dephasing rates. The Rabi coupling frequency, corresponding to half the normal mode splitting, is denoted as $\Omega_{R}$ and the complex UP and LP eigenfrequencies are given as $\tilde{\omega}_{\mathrm{UP}}$ and $\tilde{\omega}_{\mathrm{LP}}$, respectively. This expression evidently shows two resonances, at the LP and UP frequencies, respectively, occurring when the denominator is minimized, and whose positions depend on the Rabi coupling $\Omega_{R}$. In addition, it shows an antiresonance, corresponding to a minimum of the numerator, occurring in between LP and UP at the bare exciton frequency $\omega_{X}$, independently of the coupling. As seen in Fig. 3(b) (dotted red line), this coupled mode model convincingly reproduces the experimentally measured spectral phase when taking into account the values for energies of the UP and LP resonances and their dephasing rates deduced from Fig. 3(a). Based on this agreement, we can now give an intuitive interpretation for the observed antiresonance. When 

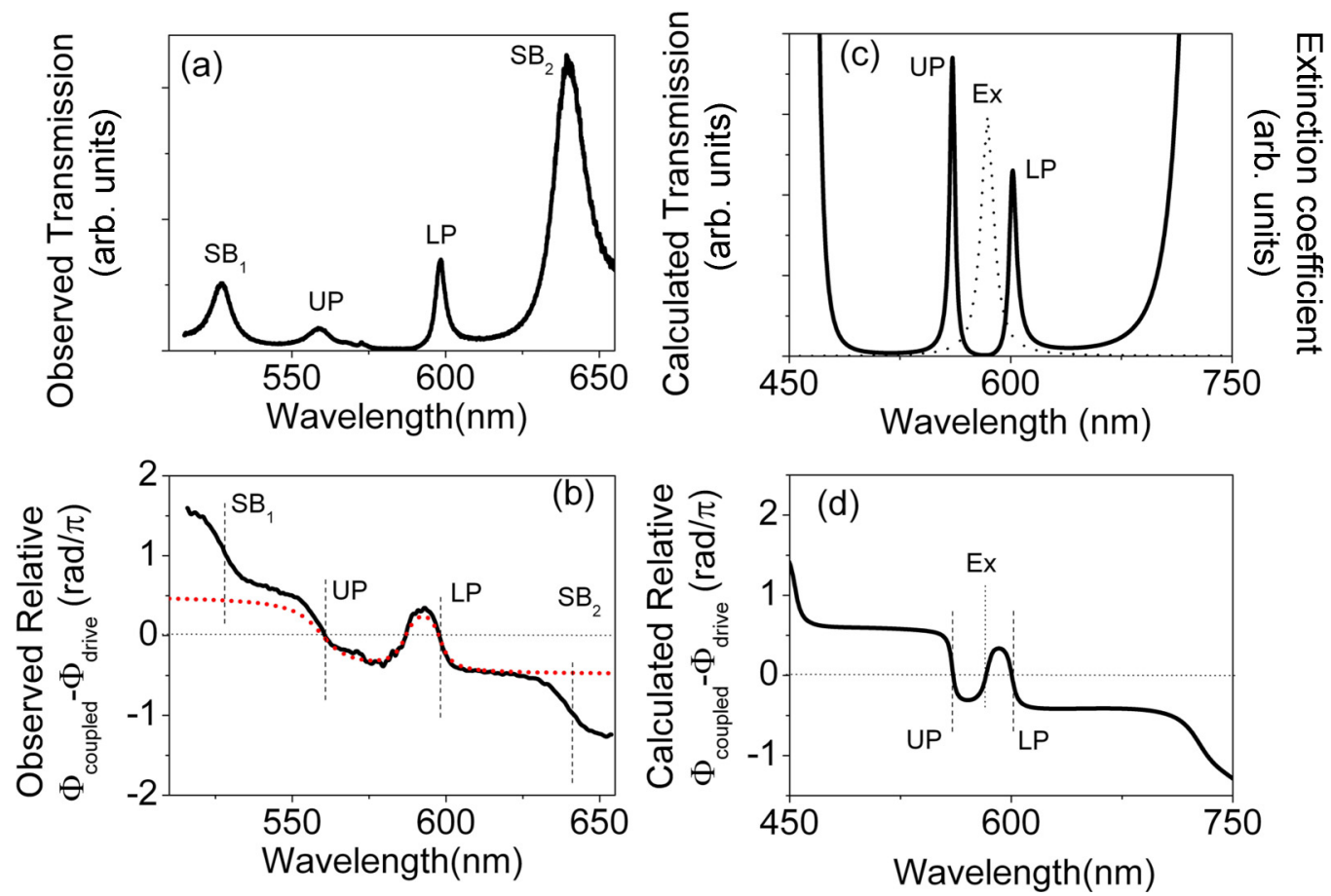

FIG. 3. (Color online) (a) Normalized experimental transmission spectrum of the microcavity at $40^{\circ}$ angle of incidence. (b) Spectral phase of the corresponding electric field extracted using SI (black solid line), together with a fit (dotted red line) described in the text. (c) Calculated transmission spectrum using TM formalism (black line). The absorption spectrum of the $J$-aggregate dye is shown as a black dotted line. (d) Spectral phase deduced by TM calculation. Phase jumps at the UP and LP resonance and the antiresonance at the exciton wavelength are shown as dashed vertical lines.

coupling laser light into the microcavity at the frequency of the uncoupled exciton resonance, the field that is reemitted by the excitons is phase shifted by $180^{\circ}$ with respect to the driving field (with a $90^{\circ}$ phase shift coming from the exciton resonance and another $90^{\circ}$ shift between the radiated field and the polarization). Destructive interference between both fields essentially cancels the cavity field and minimizes light transmission at this frequency.

This simple model suggests that the antiresonance, with the corresponding phase jump by $\approx-\pi$ for increasing frequency, always occurs at the frequency of the uncoupled exciton. The sign of the phase jump is opposite to that at the polariton resonances. To test this assertion, we repeated the SI analysis for several angles of incidence. The experimentally measured spectral phases are shown in Fig. 4(a). While the LP and UP frequencies indeed shift as a function of incidence angle [as shown in the inset of Fig. 2(a)], the antiresonance phase shift always occurs in correspondence with the exciton resonance, independently of the Rabi coupling. Antiresonance wavelengths measured for a series of incidence angles are shown in the inset of Fig 4(a).

A more quantitative simulation of the microcavity complex transmission can only be obtained with TM calculations. Figure 3(c) shows the calculated transmission spectrum and Fig. 3(d) the corresponding spectral phase at $40^{\circ}$ incidence angle. The calculations display good agreement with the experimental data, accurately reproducing the phase shifts at the polariton resonances, the antiresonance, and also the sidebands. Figure 4(b) shows the calculated relative phase shift for different incident angles, and again it is clear that the antiresonance phase shift always occurs at the exciton resonance.

We can now use the SI results to interpret the upconversion experiments reported in Fig. 2(b). As seen from the transmission spectra in Fig. 2(a), the total electric field which is up-converted by mixing with the gate pulse is given a coherent superposition of the UP and LP emission as well as the sideband emission at $640 \mathrm{~nm}$. Due to the broad spectrum of the near-infrared gate pulse, the contribution of each of the three resonances to the up-conversion signal depends sensitively on the chosen detection wavelength. UP and LP emission are in phase and hence will constructively interfere at time zero upon impulsive excitation, giving rise to pronounced maxima in the up-conversion signal at delay times of 0 fs and at integer multiples of their beat period (cosinelike oscillations). On the other hand, the sideband is phase shifted by $\pi$ with respect to both the UP and the LP resonances, so that any superposition of the LP (UP) with the sideband would give rise to destructive interference at time zero (sinelike oscillations). Based on this analysis, we can conclude that the up-conversion signal at $360 \mathrm{~nm}$ is indeed dominated by UP/LP beats, whereas the contribution of the sideband emission at this wavelength is very weak. A detailed simulation of the up-converted transmission decay, shown in Fig. 2(b) as a dashed line (see the Supplemental Material for details on the simulation [32]), clearly supports this assignment.

In conclusion, we have studied the dynamical response of an organic microcavity working in the strong-coupling regime by the combination of ultrafast up-conversion and spectral interferometry techniques. The coherent transmission 

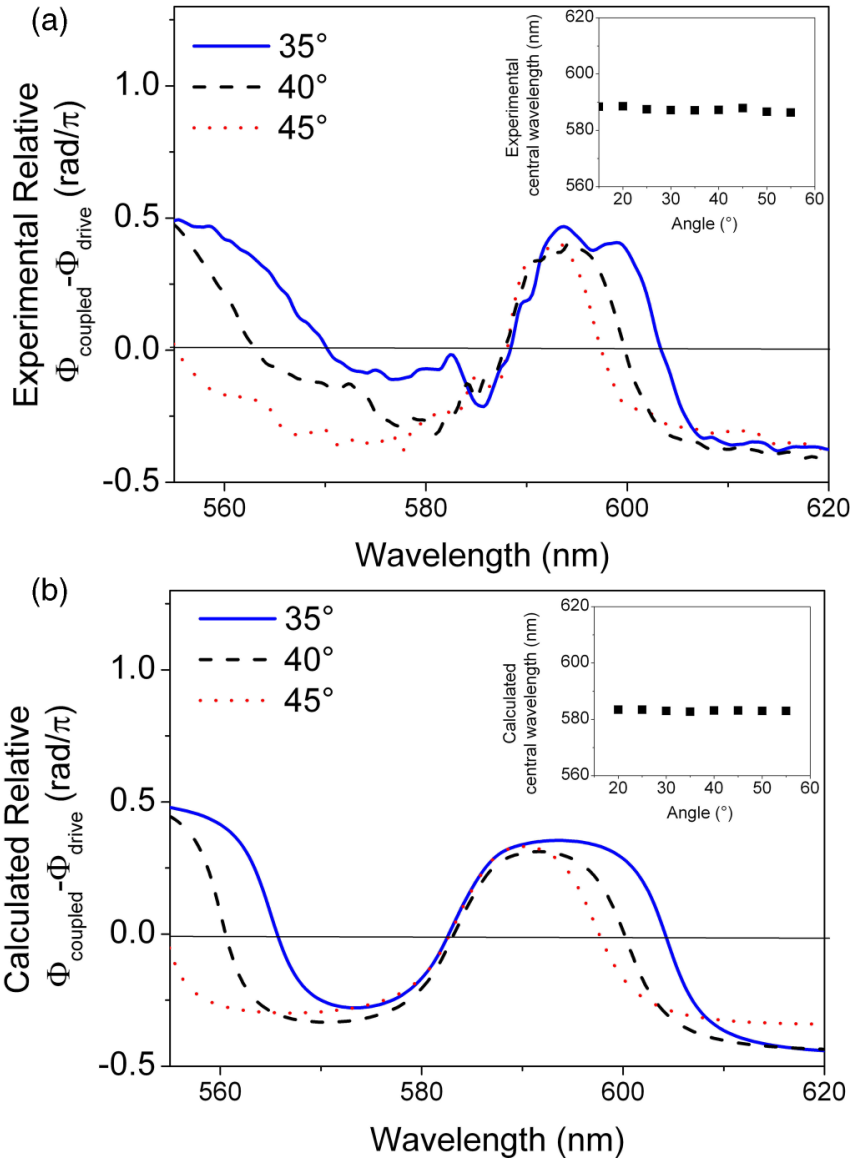

FIG. 4. (Color online) (a) Experimental and (b) calculated spectral phase shifts between the incident laser field and the field transmitted through the cavity at different incident angles. In the insets, the (a) experimental and (b) calculated center wavelengths of the antiresonance phase shift are depicted as a function of incident angle.

arising from the cavity polariton modes was time resolved, revealing 30-fs temporal oscillations. The phase of the transmitted electric field, extracted by spectral interferometry, reveals not only the anticipated polariton resonances, but also a pronounced antiresonance negative phase shift in the frequency domain, caused by destructive interference between the coherent drive and the field radiated by the $J$-aggregate exciton. This observation provides direct experimental insight into the dynamics of strong coupling in microcavities on femtosecond time scales: The incident light is coupled to a photonlike cavity mode which periodically exchanges energy with the excitonic mode until it is coupled out of the cavity resonator. More generally, our results show that precise insight into the ultrafast dynamics of energy exchange and strongcoupling phenomena can be obtained from an amplitude and phase-resolved study of linear optical spectra, without the need for advanced time-resolved nonlinear optical spectroscopy techniques. Such ultrafast, coherent coupling phenomena are ubiquitous in meso- and nanoscale systems, and we therefore believe that our findings will prove very valuable for future spectroscopic investigations of such processes, in particular, when combined with advanced, broadband scattering-type or adiabatic nanofocusing near-field spectroscopy techniques providing a spatial resolution in the $10-\mathrm{nm}$ range.

T.V., S.K.R., D.C., and D.G.L. wish to thank the UK EPSRC for funding this work via Grant No. EP/G062404/1 "Engineering polariton nonlinearity in organic and hybridsemiconductor microcavities," and the European Union, FP7 ITN project Icarus (237900). C.L. wishes to thank the Deutsche Forschungsgemeinschaft (SPP 1391, and DFG-NSF Materials World Network), the European Union, FP7 “CRONOS” (Grant No. 280879-2), and the Korea Foundation for International Cooperation of Science and Technology (Global Research Laboratory project, K20815000003) for financial support. G.C. acknowledges support by the EC under Graphene Flagship (Contract No. CNECT-ICT-604391). T.V. and G.C. thank Francesco Tassone and Salvatore Stagira for the useful discussions.
[1] R. Balili, V. Hartwell, D. Snoke, L. Pfeiffer, and K. West, Science 316, 1007 (2007).

[2] S. Kéna-Cohen and S. R. Forrest, Nat. Photonics 4, 371 (2010).

[3] C. Schneider, A. Rahimi-Iman, N. Y. Kim, J. Fischer, I. G. Savenko, M. Amthor, M. Lermer, A. Wolf, L. Worschech, V. D. Kulakovskii, I. A. Shelykh, M. Kamp, S. Reitzenstein, A. Forchel, Y. Yamamoto, and S. Höfling, Nature (London) 497, 348 (2013)

[4] A. Amo, J. Lefrère, S. Pigeon, C. Adrados, C. Ciuti, I. Carusotto, R. Houdré, E. Giacobino, and A. Bramati, Nat. Phys. 5, 805 (2009).

[5] P. G. Savvidis, J. J. Baumberg, R. M. Stevenson, M. S. Skolnick, D. M. Whittaker, and J. S. Roberts, Phys. Rev. Lett. 84, 1547 (2000).

[6] M. Saba, C. Ciuti, J. Bloch, and R. Andre, Nature (London) 414, $731(2001)$
[7] J. R. Tischler, M. S. Bradley, V. Bulović, J. H. Song, and A. Nurmikko, Phys. Rev. Lett. 95, 036401 (2005).

[8] T.-C. Lu, J.-R. Chen, S.-C. Lin, S.-W. Huang, S.-C. Wang, and Y. Yamamoto, Nano Lett. 11, 2791 (2011).

[9] H. Deng, G. Weihs, C. Santori, J. Bloch, and Y. Yamamoto, Science 298, 199 (2002).

[10] J. Kasprzak, M. Richard, S. Kundermann, A. Baas, P. Jeambrun, J. M. J. Keeling, F. M. Marchetti, M. H. Szymańska, R. André, J. L. Staehli, V. Savona, P. B. Littlewood, B. Deveaud, and L. S. Dang, Nature (London) 443, 409 (2006).

[11] O. Lyngnes, J. D. Berger, J. P. Prineas, S. Park, G. Khitrova, H. M. Gibbs, F. Jahnke, M. Kira, and S. W. Koch, Solid State Commun. 104, 297 (1997).

[12] T. B. Norris, J.-K. Rhee, C.-Y. Sung, Y. Arakawa, M. Nishioka, C. Weisbuch, Phys. Rev. B 50, 14663 (1994).

[13] G. Khitrova and H. M. Gibbs, Rev. Mod. Phys. 71, 1591 (1999). 
[14] M. Perrin, P. Senellart, A. Lemaître, and J. Bloch, Phys. Rev. B 72, 075340 (2005).

[15] F. Tassone, C. Piermarocchi, V. Savona, A. Quattropani, and P. Schwendimann, Phys. Rev. B 56, 7554 (1997).

[16] D. G. Lidzey, T. Virgili, D. D. C. Bradley, M. S. Skolnick, S. Walker, and D. M. Whittaker, Opt. Mater. (Amsterdam) 12, 243 (1999).

[17] R. F. Oulton, N. Takada, J. Koe, P. N. Stavrinou, and D. D. C. Bradley, Semicond. Sci. Technol. 18, S419 (2003).

[18] R. J. Holmes and S. R. Forrest, Org. Electron. 8, 77 (2007).

[19] J. Wenus, L. G. Connolly, and D. G. Lidzey, Phys. Status Solidi 2, 3899 (2005).

[20] M. S. Bradley, J. R. Tischler, Y. Shirasaki, and V. Bulović, Phys. Rev. B 78, 193305 (2008).

[21] S. Ceccarelli, J. Wenus, M. S. Skolnick, and D. G. Lidzey, Superlattices Microstruct. 41, 289 (2007).

[22] D. M. Coles, P. Michetti, C. Clark, A. M. Adawi, and D. G. Lidzey, Phys. Rev. B 84, 205214 (2011).

[23] J. Chovan, I. E. Perakis, S. Ceccarelli, and D. G. Lidzey, Phys. Rev. B 78, 045320 (2008).

[24] D. M. Coles, P. Michetti, C. Clark, W. C. Tsoi, A. M. Adawi, J.-S. Kim, and D. G. Lidzey, Adv. Funct. Mater. 21, 3691 (2011).

[25] V. M. Agranovich, M. Litinskaia, and D. G. Lidzey, Phys. Rev. B 67, 085311 (2003).

[26] M. Litinskaya, P. Reineker, and V. M. Agranovich, J. Lumin. 110, 364 (2004).

[27] P. Michetti and G. C. La Rocca, Phys. Rev. B 71, 115320 (2005).

[28] T. Virgili, D. Coles, A. M. Adawi, C. Clark, P. Michetti, S. K. Rajendran, D. Brida, D. Polli, G. Cerullo, and D. G. Lidzey, Phys. Rev. B 83, 245309 (2011).
[29] T. Schwartz, J. A. Hutchison, J. Léonard, C. Genet, S. Haacke, and T. W. Ebbesen, ChemPhysChem 14, 125 (2013).

[30] W. Wang, P. Vasa, R. Pomraenke, R. Vogelgesang, A. De Sio, E. Sommer, M. Maiuri, C. Manzoni, G. Cerullo, and C. Lienau, ACS Nano 8, 1056 (2014).

[31] P. Vasa, W. Wang, R. Pomraenke, M. Lammers, M. Maiuri, C. Manzoni, G. Cerullo, and C. Lienau, Nat. Photonics 7, 128 (2013).

[32] See Supplemental Material at http://link.aps.org/supplemental/ 10.1103/PhysRevB.91.201305 for details on J-aggregate microcavity fabrication, transfer-matrix modeling of the microcavity transmission, time-resolved up-conversion experiment, simulations of the up-converted transmission decay and quantum derivation of the transmission coefficient of a microcavity.

[33] E. W. Knapp, Chem. Phys. 85, 73 (1984).

[34] J. R. Tischler, M. Scott Bradley, Q. Zhang, T. Atay, A. Nurmikko, and V. Bulović, Org. Electron. 8, 94 (2007).

[35] G. Bongiovanni, A. Mura, F. Quochi, S. Gürtler, J. L. Staehli, F. Tassone, R. P. Stanley, U. Oesterle, and R. Houdré, Phys. Rev. B 55, 7084 (1997).

[36] A. D. Jameson, J. L. Tomaino, S.-Y. Lee, G. Khitrova, H. M. Gibbs, C. N. Böttge, A. C. Klettke, M. Kira, and S. W. Koch, Optica 1, 276 (2014).

[37] L. Lepetit, G. Chériaux, and M. Joffre, J. Opt. Soc. Am. B 12, 2467 (1995).

[38] C. Ropers, T. Elsaesser, G. Cerullo, M. Zavelani-Rossi, and C. Lienau, New J. Phys. 9, 397 (2007).

[39] C. Sames, H. Chibani, C. Hamsen, P. A. Altin, T. Wilk, and G. Rempe, Phys. Rev. Lett. 112, 043601 (2014). 